Activation of Catalysts in Olefin Reactions

THE observations recorded by Evans et al. ${ }^{1}$ establish that boron trifluoride alone cannot cause the polymerization of $i s o$-butene or of di-iso-butene unless some unidentified promoter is present. It is
the intention of this letter to direct attention to a number of similar observations affecting olefin reactions.

Ethylene is not polymerized by pure aluminium chloride at temperatures below $50^{\circ}$ unless traces of hydrogen chloride or moisture are present $^{2}$. The reaction of olefins with iso-paraffins and with aromatic hydrocarbons under the influence of aluminium chloride and certain other inorganic chlorides is promoted by hydrogen chloride ${ }^{3}$. Similarly, boron trifluoride will not eatalyse reaction between an olefin and an iso-paraffin unless small amounts of water or hydrogen fluoride and a finely divided metal are present.

If hydrogen halide is the common promoter in all these reactions, we may assume that its primary action is the same in each. The most promising suggestion is that olefin acts as a proton acceptor. The function of the catalyst is to cause reaction between a charged olefin addendum and the other reactant. This hypothesis has the advantage of providing a common explanation of the catalytic action of inorganic halides and also of hydrogen fluoride and of sulphuric and phosphoric acids in bringing about all the reactions mentioned above.

The University,

Birmingham, 15 .

1 Evans, Holden, Plesch, Polanyi, Skinner and Weinberger, Nature, 157, 102 (1946)

2 Ipatieff and Grosse, J. Amer. Chem. Soc., 58, 915 (1936).

"Ipatieff, "Catalytic Reactions at High Pressures and Temperatures",

pp. 697, 701.
4 Ipatieff, J. Amer. Chem. Soc., 57, 1616 (1935).

WE regret to have left unmentioned the facts referred to by Dr. Hickinbottom. They may have some bearing on our findings, but it is too early to discuss this at length. We may mention, however, that the action of titanium chloride (Ticl, ) on isobutene at low temperature which we found to be induced by traces of water is not affected by which we found to be
dry hydrogen chloride.

The University,

M. POLANYi.

Manchester, 13.

\section{A Dropping Mercury Electrode of Improved Sensitivity}

Lingane and Loveridge ${ }^{1}$ have recently emphasized the desirability of using a capillary electrode with drop-time between one and six seconds in polarographic work. For a given tube, the drop-time ( $\tau$ ) is inversely proportional to the rate of flow of mercury $(m)$, which may dimensions by altering the height of the mercury reservoir or the proportional to $m^{2 / 3} \tau^{1 / 8}$, or, in terms of the rate of increase of surface area of the drop $\left(\frac{d A}{d t}\right)$, to $\tau^{1 / 2} \frac{d A}{d t}$. If the rate of flow of mercury is increased sufficiently, the mercury no longer issues from the orifice in discrete drops, but in a fine stream. It has been possible, however, by constructing a capillary tube with oriflce larger than the bore higher in the tube, to use an increased rate of flow of mercury without simultaneously increasing the drop-time.

The theoretical equation for the sensitivity of a dropping mercury electrode, as given first by llkovic ${ }^{2}$, is

$$
\frac{i_{D}}{c}=k n D^{1 / 2} m^{2 / 3} \tau^{1 / 8} \quad \text {. . . }
$$

where $i_{D}$ is average diffusion current during life of drop; $n$ is number of electrons per mole involved in reduction ; $D$ is diffusion coefficient of reducible substance; $c$ is concentration of reducible substance $m$ is rate of flow of mercury ; $\tau$ is drop-time.

Kolthoff and Kahan ${ }^{3}$ have shown that, in decinormal potassium chloride solution, the mercury drop just before it falls is suspended from a narrow neck of mercury, and is not attached to the glass of the capillary. In considering the relation between drop-time and $\begin{aligned} & \text { dimensions of the capillary, therefore, we may use Tate's equation } \\ & M g\end{aligned}=2 \pi r_{0} T$, or the modiflation adapted by Hawkins and Brown

$$
m \tau g \frac{\left(\rho_{\mathrm{Hg}}=\rho_{\mathrm{g}}\right)}{\rho_{\mathrm{Hg}}}=\varphi r_{0} T
$$

where $r_{0}$ is radius of capillary at orifice; $T$ is interfacial tension $\rho \mathrm{Hg}, \rho_{\mathrm{g}}$ are densities of mercury and solution respectively; $\varphi$ is a function of $r_{0} / V^{1 / 3}$ which varies with the ratio of radius of capillary and radius of drop.

Equation (2) has been tested for the dropping mercury electrode using the experimental data from Maas $^{5}$ and from Muller ${ }^{6}$, selectin 380 dynes $\mathrm{cm} .^{-1}$ for the interfacial tension of mercury in decinorma potassium chloride. It will be seen that, for a given capillary immersed in a given solution at constant temperature, $m \tau$ is approximately constant, and the value $\mathrm{cf}|\varphi|$ is much closer to $2 \pi$ than has been reported for other liquids.

Data from Maas $^{5}$

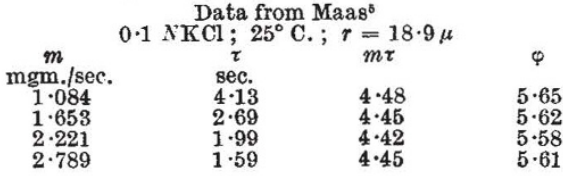

Data from Muller

$m$
mgm./sec.
$2 \cdot 56$
$2 \cdot 19$
$1 \cdot 82$
$1 \cdot 44$
$1 \cdot 06$

$0 \cdot 1 \mathrm{NKNO}_{3} ; r=27 \mu$

$\begin{array}{ccc}\boldsymbol{\tau} & m \boldsymbol{r} & \varphi \\ \text { sec. } & & \\ 2 \cdot 64 & 6 \cdot 76 & 5 \cdot 99 \\ 3 \cdot 04 & 6 \cdot 62 & 5 \cdot 87 \\ 3 \cdot 66 & 6 \cdot 66 & 5 \cdot 90 \\ 4 \cdot 63 & 6 \cdot 67 & 5 \cdot 91 \\ 6 \cdot 27 & 6 \cdot 65 & 5.90\end{array}$

Although mercury does not wet glass, the rate of flow from the dropping mercury electrode follows Poiseuille's equation ${ }^{5}$, provided that the pressure is corrected for the back pressure caused by the surface tension of the expanded drop, and also the hydrostatic pressure when the capillary is immersed in a solution. The kinetic energy of the issuing mercury is very small $(\sim 0.1$ per cent of the potential energy of the corresponding mass of mercury at the height of the
reservoir), and may be neglected.

$$
\frac{V}{\tau}=\frac{\pi P r_{c}{ }^{4}}{8 \eta l}
$$

where $\boldsymbol{V}$ is volume of single drop; $P$ is corrected pressure; $\eta$ is viscosity coefficient of mercury ; $r_{c}$ is radius of capillary; $l$ is length

From equations (2) and (3) we have

$$
\frac{\pi P r_{c}^{4}}{8 \eta l} g \rho^{\prime} \tau=\varphi r_{0} T
$$

Substituting in equation (1),

$$
\frac{i_{D}}{c}=k^{\prime} n D^{1 / 2} \frac{T^{1 / 6}}{\eta^{1 / 2}} \frac{P^{1 / 2} r_{c}^{2} r_{0}^{1 / 6}}{l^{1 / 2}}
$$

Thus, in a given solution at any flxed temperature, with $n, D, \rho, T, \eta$ all constant, $\tau$ will be proportional to $\frac{l}{\ddot{P}} \frac{r_{0}}{r_{c}{ }^{4}} ; \frac{i_{D}}{c}$ will be proportional to $\frac{P^{1 / 2}}{1 / 2} r_{0}^{1 / 6} r_{c}^{2}$.

For a uniform capillary, $r_{0}=r_{c}=r$,

$\left.\begin{array}{l}\tau \text { will be proportional to } 1 / r^{s} \\ \frac{i_{D}}{c} \text { will be proportional to } r^{13 / 6}\end{array}\right\}$ with $P, l$ constant.

If the drop-time is to have a value between 1 and 6 sec., the radius of a uniform capillary may be varied only by a factor 1.82 , the polarographic sensitivity varying at the same time by a factor $3 \cdot 65$.

It is possible to increase the sensitivity of a capillary without changing $P$ and $l$ (where $l$ is now the effective length consistent with the application of Poiseuille's equation), if it is arranged that the radius at the oriflce is greater than the radius higher in the tube. The drop-time remains constant if $r_{0} / r_{c}^{4}$ is kept constant, so that although there is a much faster flow of mercury through the tube the mercury is discharged in large discrete drops. With $r_{0}$ greater than $r_{c}$ mercury is discharged in large discrete drops. With $r_{0}$ greater than $r_{c}$ and $r_{0} / r_{c}^{6}$ constant,

Thus, if the radius of the capillary is doubled, the radius of the oriflce Thus, if the radius of the capillary is doubled, the radius of the orifice must be increased sixteen times to maintain a constant drop-time. The sensitivity (or rate of increase of surface
6.35 times without any variation of $P$ or $l$.

This special type of capillary may be constructed by fusing together capillaries of the required dimensions, or empirically by cutting of a pulled thermometer capillary just beyond its narrowest part. One such tube had the constants $m=6.5 \mathrm{mgm}$. $/ \mathrm{sec}$., $\tau=3.7 \mathrm{sec}$., $m^{2 / 8} \tau^{1 / 6}=4 \cdot 3 \mathrm{mgm}^{2 / 3}$ sec. $^{-1 / 2}$, and functioned satisfactorily in the polarographic circuit for more than a year. The expected increase in polarographic sensitivity was obtained, but the residual current was so large as to preclude the use of the highest galvanometer sensitivities. This phenomenon must be a scribed to the increased condenser the drop surface. For a uniform interfacial charge distribution,

$$
i_{c}=K \psi \frac{d A}{d t}=K^{\prime} \psi m^{2 / 3} \tau^{-1 / 3},
$$

where $K$ is average capacity of double layer per unit area; $\psi$ is poten. tial difference between mercury surface and solution.

The slope of the condenser current curve will be proportional to the rate at which fresh surface is exposed, that is, to $\tau_{c}^{4} / r_{0}^{1 / 8}$. If $r_{c}$ is doubled with $\tau$ held constant, the slope will be increased 6.35 times. Moreover, the stirring of the solution, induced by the rapid formation of large mercury drops, mav lead to acceleration of transfer of reducible substances to the mercury surface. Lingane and Loveridge ${ }^{1}$ have already reported a deviation from linearity between $\frac{i_{D}}{c}$ and $m^{2 / 8} \tau^{1 / 4}$ for rapidly dropping capillaries.

It is thus probable that the new type of capillary will be useful in special applications rather than in routine polarographic analysis.

This work was done as a member of a team flnanced by the Agricultural Research Council to work on the diagnosis and cure of mineral deficiencies in plants.

East Malling Research Station,

J. P. R. RICHES.

\section{Botany School,}

Cambridge.

${ }^{1}$ Lingane and Loveridge, $J$. Amer. Chem. Soc., 66, 1425 (1944).

Ilkovic Coll Crech Chem. Comm 6, 498 (1934)

' Ilkovic, Coll. Czech. Chem. Comm., 6, 498 (1934).

Kolthoff and Kahan, $J$. Amer. Chem. Soc., 64, 2553 (1942).

s Hawkins and Brown, $J$.Amer. Chem. Soc., 41, 499 (1919).

Muller, J. Amer. Chem. Soc., 66, 1019 (1944). 